\title{
Endoscopic ultrasound diagnosis of colon cancer metastatic to the pancreas
}

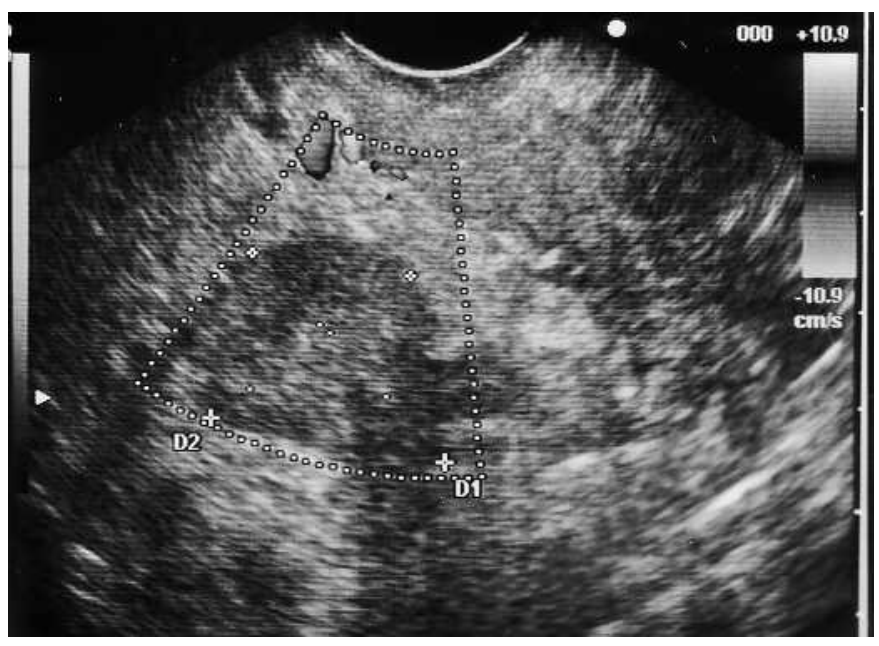

Figure 1 Mass in the head of the pancreas, seen at endoscopic ultrasound (7.5-MHz curvilinear). The lesion is hypodense and of mixed echotexture, and appears to have pseudopods extending into the pancreatic head.

A 53-year-old woman presented with a history of abdominal pain, nausea, vomiting, and a 40-kg weight loss, without change in bowel pattern. Physical examination revealed epigastric tenderness, good bowel sounds, and an abdominal fluid wave. Rectal examination was normal without occult blood. Abdominal computed tomography (CT) scan revealed a hypodense area of enlargement in the head of the pancreas, ascites, and multiple metastatic lesions in the liver, and normal bowels.

Endoscopic ultrasound (EUS) was performed to evaluate the pancreas. In the head of the gland there was a lobular and irregular hypoechogenic structure measuring $25 \times 28 \mathrm{~mm}$ (Figure $\mathbf{1}$ ). The lesion also had what appeared to be pseudopods extending into the pancreatic head. EUSguided fine-needle aspiration was performed. Cytologic evaluation demonstrated adenocarcinoma. The tissue stained positive for CK20 and negative for CK7 and showed uniform immunoreactivity with CDX2. These results strongly suggested a primary colorectal cancer and not a primary pancreatic adenocarcinoma.

Colonoscopy revealed a large, partially obstructing mass in the ascending colon, just above the ileocecal fold (Figure 2). The patient was offered a palliative partial colectomy or placement of a colonic stent. The patient did not wish to pursue surgery, and a $22 \times 90-\mathrm{mm}$ colonic self-expanding metal stent (Boston Scientific, Natick, Massachusetts, USA) was placed across the stricture, with the proximal end of the stent in the cecum. Her bowel remained patent until her death 2 months later.

Reports of colon cancer metastasizing to the pancreas are very uncommon [1]. Immunohistochemically, the CK7-/CK20+ phenotype seen here predicts colorectal origin with considerable accuracy and independently of other clinical information [2]. CDX2 stains homogeneously in tissue arising from the colon (as was seen here) or duodenum and heterogeneously in pancreatic adenocarcinoma [3]. This is only the third report of EUS detection of colorectal cancer metastasis to the pancreas [4.5].

Endoscopy_UCTN_Code_CCL_1AF_2AZ_3AB

\section{R. Rengen', J. De ${ }^{2}$, M. M. Kott ${ }^{2}$, D. G. Adler ${ }^{\mathbf{1}}$}

${ }^{1}$ Department of Internal Medicine, Division of Gastroenterology and Hepatology, University of Texas-Houston Health Science Center, Houston, Texas, USA

2 Department of Pathology, University of Texas-Houston Health Science Center, Houston, Texas, USA.



Figure 2 Endoscopic appearance of colonic malignancy seen in the right colon, immediately distal to the ileocecal fold.

\section{References}

${ }^{1}$ Z'graggen K, Fernandez-del Castillo C, Rattner DW et al. Metastases to the pancreas and their surgical extirpation. Arch Surg 1998; 133: 413-418

2 Tot T, Samii S. The clinical relevance of cytokeratin phenotyping in needle biopsy of liver metastasis. APMIS 2003; 111: 1075-1082

3 Werling RW, Yaziji H, Bacchi CE, Gown AM. CDX2, a highly sensitive and specific marker of adenocarcinomas of intestinal origin: an immunohistochemical survey of 476 primary and metastatic carcinomas. Am J Surg Pathol 2003; 27: 303-310

${ }^{4}$ DeWitt J, Jowell P, Leblanc J et al. EUS-guided FNA of pancreatic metastases: a multicenter experience. Gastrointest Endosc 2005; 61: 689-696

${ }^{5}$ Fritscher-Ravens A, Sriram PV, Krause C et al. Detection of pancreatic metastases by EUSguided fine-needle aspiration. Gastrointest Endosc 2001; 53: $65-70$

\section{Corresponding author}

\section{G. Adler, M.D.}

Division of Gastroenterology and Hepatology University of Texas-Houston Health Science Center, MSB 4.234, 6431 Fannin, Houston, Texas 77030, USA

Fax: +1-713-500-6699

E-mail: douglas.adler@uth.tmc.edu 